\title{
ENFOQUE PARA LA GESTIÓN DE PROCESOS DE NEGOCIO SEMÁNTICOS UTILIZANDO ONTOLOGÍAS
}

\author{
Edgar Tello-Leal \\ Universidad Autónoma de \\ Tamaulipas \\ etello@uat.edu.mx
}

\author{
Jorge Alejandro Carreón \\ Universidad Autónoma de \\ Tamaulipas \\ jacarreon@uat.edu.mx
}

\author{
Mariby Lucio Castillo \\ Universidad Autónoma de \\ Tamaulipas \\ mlucio@uat.edu.mx
}

(Tipo de Artículo: Revisión. Recibido el 22/05/2013. Aprobado el 29/06/2013)

\begin{abstract}
RESUMEN
Las oportunidades de mercado actuales y los avances en las Tecnologías de Información y Comunicaciones, alientan a las organizaciones a establecer redes de colaboración. Las redes de colaboración consisten en relaciones entre organizaciones por un período de tiempo determinado para alcanzar objetivos de negocio comunes, lo cual implica una integración orientada a procesos entre organizaciones heterogéneas y autónomas, que debe ser alcanzada tanto en una perspectiva de negocio como en una perspectiva de implementación. La Gestión de Procesos de Negocio Semánticos es un enfoque que permite incrementar el nivel de automatización mediante el uso de ontologías y servicios Web, lo cual permite enfrentar los retos actuales de la Gestión de Procesos de Negocio, tales como descubrimiento de servicios, reutilización de procesos, y análisis de los procesos ejecutados. En este trabajo de investigación se presenta el diseño e implementación de un modelo de procesos de negocio, el cual es modelado usando el lenguaje BPMN y anotado semánticamente con conceptos de la ontología BPMO, permitiendo llevar a cabo el descubrimiento de servicios Web, fragmentación de procesos y reutilización de procesos, así como consultas sobre las instancias del modelo de proceso generadas mediante un razonador automático.
\end{abstract}

Palabras clave

BPMN, Gestión de procesos de negocio, proceso de negocio, Servicios Web Semánticos, WSMO.

\section{Approach for Semantic Business Process Management using Ontologies}

\begin{abstract}
Current market opportunities and the growth of Information and communication technologies encourage organizations to establish collaborative networks. The collaborative networks consist of relationships between companies during a period of time to achieve common business goals, implying a process-oriented integration between heterogeneous and autonomous companies, which must be achieved both for a business perspective and for an implementation perspective. Semantic business process management is an approach to increase the level of automation through the use of semantic technologies (like ontologies) and Web services, which allows facing some current challenges of the Business Process Management, such as service discovery, process reuse, and analysis of executed processes. In this paper we present the design and implementation of a business process model, which is modeled using the BPMN language and semantically annotated with concepts of the BPMO ontology, enabling semantic-based discovery, process fragments, and reuse of existing process artifacts, as well as queries on instances of the process model produced using automated reasoning.
\end{abstract}

Keywords

BPMN, business process, Business Process Management, Semantic Web Services, WSMO.

\section{APPROCHE Á LA GESTION DES PROCESSUS MÉTIERS SÉMANTIQUES EN UTILISANT DES ONTOLOGIES}

\begin{abstract}
RÉSUMÉ
Les opportunités de marché actuel et les progrès réussis dans les Technologies de l'Information et de la Communication, stimulent la création de réseaux de collaboration. Les réseaux de collaboration sont des relations entre les entreprises pendant un temps précis pour réussir des objectifs métiers en commun, ce qu'implique une intégration oriente vers les processus entre entreprises hétérogènes et autonomes, telle intégration doit être réussi dans un point de vue métier ainsi que dans une perspective d'implémentation. La gestion des processus métiers sémantiques est un approche qui permet d'accroitre le niveau d'automatisation au moyen de l'usage d'ontologies et services Web, ce qui permet d'affronter les défis actuels de la gestion des processus métiers, tels que la découverte de services, réutilisation des processus et l'analyse des processus réalisés. Dans ce travail de recherche on présente la conception et implémentation d'un modèle de processus métiers qui est modelé en utilisant le langage BPMN et qui est annoté sémantiquement avec des concepts de l'ontologie BPMO, en permettant réaliser la découverte des services Web, la fragmentation et la réutilisation des processus, de même que des consultations au sujet des instances du modèle de processus créés au moyen d'un raisonneur automatique.
\end{abstract}

\section{Mots-clés}

BPMN, gestion des processus métiers, processus métiers, services Web sémantiques, WSMO. 


\section{INTRODUCCIÓN}

La Gestión de Procesos de Negocio (BPM, del inglés Business Process Management) ha recibido una considerable atención en los últimos años tanto de la comunidad de administración de negocios como de ciencias de la computación. En donde un proceso de negocio consiste en un conjunto de actividades que se realizan coordinadamente en un entorno organizacional y técnico [1]. Estas actividades se realizan conjuntamente con una meta de negocio. Cada proceso de negocio es ejecutado por una organización, pero estos procesos pueden interactuar con los procesos de negocio ejecutados por otras organizaciones o unidades de negocio.

El enfoque BPM incluye conceptos, métodos y técnicas para soportar el diseño, administración, configuración, ejecución y análisis de los procesos de negocio [1]. Las Tecnologías de Información, en general, y los Sistemas de Información, en particular, desempeñan un rol muy importante en la gestión de procesos de negocio, debido a que una gran parte de las actividades que una organización ejecuta son soportadas por los sistemas de información. Sin embargo, los principales retos a los que se enfrenta la BPM actualmente, son permitir un análisis de los procesos de negocio ejecutados en forma automática, así como la reutilización de procesos de negocio ejecutados $\mathrm{y} / \mathrm{o}$ predefinidos por una organización, con el objetivo de mejorar la toma de decisiones y disminuir los costos y tiempos de transacción.

Con el fin de hacer frente a estos retos surge un enfoque de Gestión de Procesos de Negocio Semánticos (SBPM, del inglés Semantic Business Process Management) a través del cual se intenta incrementar el nivel de automatización de la BPM mediante el uso de lenguajes ontológicos y servicios Web semánticos para la representación tanto de la perspectiva de negocio como la perspectiva de implementación de un proceso de negocio [2]. El objetivo de SBPM es habilitar la transformación entre el nivel o perspectiva de negocio a nivel de implementación a través de la aplicación de razonamiento automático, facilitando el descubrimiento de procesos, fragmentos de procesos, y/o composición de procesos.

En este trabajo de investigación se presenta una implementación de un enfoque SBPM utilizando los conceptos de la ontología BPMO [3], lo que conlleva un mayor nivel de automatización en cada una de las fases del ciclo de vida BPM, derivando funcionalidades que permiten el descubrimiento de SWS, composición de procesos y reutilización de procesos de negocio. Primeramente, el proceso de negocio es modelado conceptualmente usando el lenguaje BPMN [4], a continuación, en cada elemento del proceso de negocio se realiza la anotación semántica usando los conceptos de la ontología, lo cual posibilita realizar consultas sobre las instancias del modelo de proceso de negocio generado, utilizando un razonador automático.
Además, se muestran la instancias del proceso negocio generadas en forma automática basadas en WSML [5], [6].

\section{GESTIÓN DE PROCESOS DE NEGOCIO Y TECNOLOGÍAS SEMÁNTICAS}

\subsection{Gestión de procesos de negocio semánticos}

El objetivo de SBPM es el combinar BPM con tecnologías semánticas, en particular ontologías y servicios Web semánticos (SWS, del inglés Semantic Web Services) lo cual permitirá alcanzar un mayor nivel de automatización en el ciclo de vida de BPM y proveer funciones y/o recursos que ayuden a los analistas de negocio y a los ingenieros de TI en la definición e implementación de un proceso de negocio [7].

La utilización de tecnologías semánticas en el ciclo de vida de BPM no afecta sus fases, al contrario, posibilita incrementar el grado de automatización de cada una de las fases, agregando nuevas funcionalidades 0 fortaleciendo las existentes en los sistemas de gestión de procesos de negocio [8]. El ciclo de vida de la SBPM propuesto en [8], tal como se muestra en la Fig. 1, contiene las siguientes fases:

- La fase de modelado de procesos de negocio semánticos presenta las funcionalidades de anotación semántica de procesos, autocompletado de procesos, y re-utilización de fragmentos de procesos.

- La fase de implementación de procesos de negocio semánticos incluye las funcionalidades de descubrimiento de SWS y composición de procesos.

- La fase de ejecución de procesos de negocio semánticos ofrece las funcionalidades de descubrimiento SWS dinámico e invocación SWS.

- En la fase de análisis de procesos de negocio semánticos se realizan funcionalidades de monitoreo de los procesos de negocio semánticos y minería de procesos de negocio semánticos.

Las fases que componen el ciclo de vida de la SBPM se describen en las siguientes sub-secciones con mayor detalle.

\subsubsection{Modelado de procesos de negocio semánticos}

En la primera fase del ciclo de vida de la SBPM se generan modelos de procesos de negocio anotados semánticamente. La meta de una anotación semántica es especificar explícitamente la semántica de los elementos de tipo tarea y de los elementos de decisión contenidos en el flujo de un proceso. Esta anotación debe hacer referencia a conceptos contenidos en una ontología. Mediante la anotación semántica se habilita el descubrimiento semántico en forma automática, el cual puede ser explotado en otras fases del ciclo de 
vida de SBPM, habilitando la realización de búsquedas automáticas para SWS, con lo cual se pueden implementar tareas dentro de un procesos o buscar fragmentos de procesos similares, permitiendo la reutilización de artefactos de procesos de negocio existentes [8].

Los fragmentos de proceso son partes de un modelo de proceso de negocio que se han identificado como potencialmente reutilizables [8]. El analista de negocios puede seleccionar partes de un modelo de proceso de negocio semántico y grabar estas partes como fragmentos de proceso en un repositorio de procesos de negocios semánticos habilitando su reutilización. Entonces, durante la fase de modelado de un proceso, el analista de negocio puede realizar búsquedas de fragmentos de procesos, con lo cual puede disminuir los tiempos de desarrollo y los posibles errores en el modelado.

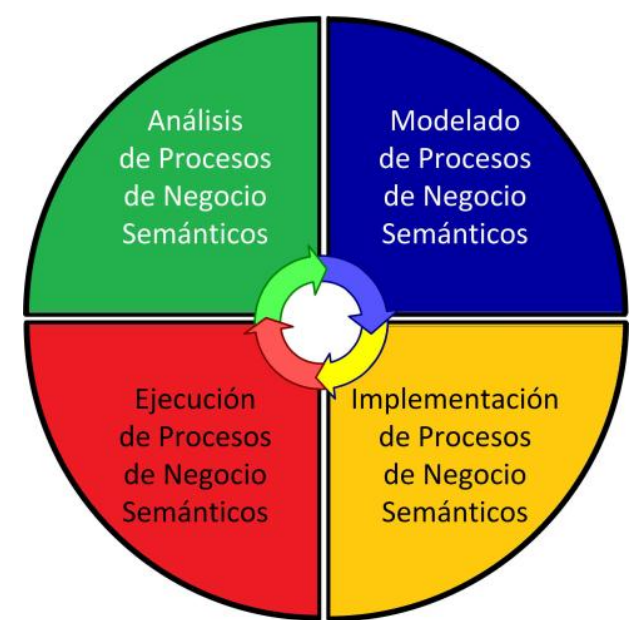

Fig. 1. Ciclo de vida de la gestión de procesos de negocio semánticos (basado en la propuesta presentada en [8])

\subsubsection{Implementación de procesos de negocio semánticos}

En esta fase, el modelo de proceso de negocio semántico es transformado en un modelo de proceso ejecutable, el cual puede ser implementado (ejecutado) mediante un motor de procesos [8], [3]. La anotación semántica del modelo de proceso de negocio realizada en la fase de modelado posibilita una mayor automatización en la fase de implementación. Mediante la anotación ontológica de la tareas contenidas en el proceso de negocio, los servicios Web semánticos adecuados para esas tareas, se pueden descubrir automáticamente. Estos servicios Web semánticos son almacenados en repositorios SWS.

\subsubsection{Ejecución del proceso de negocio semántico}

En esta fase, el modelo de proceso semántico es desplegado mediante un motor de procesos. Después de esto, el modelo estará listo para su instanciación y ejecución [8]. Por otro lado, el modelo es publicado como un servicio Web Semántico y por lo tanto es accesible para otros usuarios. El servicio Web
Semántico generado es punto de entrada para interactuar con el modelo de proceso de negocio y consumir sus funcionalidades. Además, el modelo de proceso de negocio utiliza otros SWS para implementar sus tareas, tal como se describió previamente.

La principal ventaja de utilizar SWS en la fase de ejecución es el enlace a una funcionalidad de un servicio dinámico. Los servicios que han de ser invocados fuera del proceso de negocio se pueden determinar en tiempo de ejecución mediante la infraestructura SWS usando descubrimiento basado en la semántica y después pueden enlazarse a las tareas del proceso a través de las cuales se implementarán.

\subsubsection{Análisis de procesos de negocio semánticos}

En la fase de análisis de procesos de negocio semánticos se distinguen dos funciones: el monitoreo de procesos y la minería de procesos [8]. El monitoreo de procesos tiene como objetivo proporcionar información relevante sobre la ejecución de las instancias del proceso de negocio generadas en la fase de ejecución del proceso de negocio semántico. La función de minería de procesos analiza las instancias de los procesos ya ejecutados, con el fin de detectar puntos de mejora para el modelo del proceso de negocio.

Tanto el monitoreo de procesos como la minería de procesos operan sobre el registro de eventos (logs del sistema), el cual es generado por el motor de proceso durante la ejecución del modelo de proceso de negocio. En la fase de análisis se puede habilitar una clasificación formal de los eventos de acuerdo a tipos de eventos predefinidos, lo cual puede ser enriquecido mediante una ontología de eventos.

\subsection{Servicios Web Semánticos}

Con el surgimiento de SOA y de los Servicios Web como el paradigma dominante para la fabricación de componentes funcionales de software de forma estandarizada, uno de los retos o problemas es cómo encontrar de forma eficiente los servicios Web almacenados en grandes repositorios [6]. Con el fin de encontrar una solución, una área de la investigación se centró en el uso de tecnologías semánticas para facilitar el manejo de servicios Web. El resultado de estos esfuerzos se conoce como Servicios Web Semánticos (SWS) [6], [9], en donde una interfaz de servicio Web se puede describir semánticamente a fin de permitir que una máquina de razonamiento automático pueda ser ejecutada, permitiendo el descubrimiento de componentes. De hecho, los SWS apuntan a una tecnología integrada para una nueva generación de la Web mediante la combinación de las tecnologías de Web semántica y servicios Web, lo cual convierte a Internet desde un repositorio de información hasta un sistema mundial para la computación distribuida Web. Existen múltiples iniciativas para SWS, dentro de las cuales se pueden mencionar OWL-S [10], 
WSMO [11], y SAWSDL [12] como los enfoques más importantes.

WSMO (del inglés Web Service Modeling Ontology) [11] tiene como objetivo describir todos los aspectos relacionados con los servicios generales que son accesibles a través de una interfaz de servicios Web con el objetivo final de habilitar la automatización total o parcial de las tareas (descubrimiento, selección, composición, mediación, ejecución, y monitoreo) involucradas en la integración de servicios Web. WSMO se basa conceptualmente en WSMF (del inglés, Web Service Modeling Framework) [13], presentando una refinación y extensión de este framework, además de integrar el desarrollo de una ontología formal y un conjunto de lenguajes.

Por lo tanto, WSMO proporciona especificaciones ontológicas de los elementos centrales de SWS. En framework WSMO se identifican cuatro elementos de nivel superior como conceptos principales que deben ser descritos con el fin de definir Servicios Web Semánticos:

- Ontologías. Proveen la terminología usada por otros elementos WSMO para describir aspectos relevantes del dominio de discurso. Las ontologías pueden proveer definiciones formales que son procesables por una máquina, con lo cual puede habilitar que otros componentes y aplicaciones pueden tomar decisiones en base a su significado y sentido en un contexto dado.

- Servicios Web. Representan entidades computacionales capaces de proporcionar el acceso a servicios que, a su vez, proveen un valor en un dominio. Las descripciones de servicios Web contienen las capacidades, interfaces y funcionamiento interno del servicio. En WSMO, los Servicios Web modelan los aspectos funcionales, no funcionales, y de comportamiento, que deben ser descritos semánticamente para posibilitar su uso automatizado o semi-automatizado. Cada Servicio Web representa una entidad atómica funcional que puede ser re-utilizada para construir otro componente.

- Metas. Describen aspectos relacionados a los deseos del usuario con respecto a una funcionalidad requerida. En este caso, una meta estará relacionada con aspectos del objetivo o meta de negocio que puede ser alcanzada mediante la ejecución del proceso de negocio.

- Mediadores. Describen los elementos que se encargan de los problemas de interoperabilidad entre los diferentes elementos de WSMO. Los mediadores son un concepto central para resolver las incompatibilidades en los niveles de datos, de proceso, y de protocolo.

\subsection{Ontología para el Modelado de Procesos de Negocio}

Actualmente, los analistas de negocios utilizan para el modelado de procesos de negocios notaciones basadas en BPMN (del inglés, Business Process Model and Notation) [4] y EPCs (del inglés, Event-driven Process Chains) [14] principalmente, estos lenguajes permiten crear y analizar diagramas de procesos de negocio como parte del conjunto de herramientas para BPM. Estas anotaciones son útiles en el nivel o perspectiva de negocio, proporcionando únicamente elementos gráficos con información textual muy limitada e información en relación a la semántica formal.

La Ontología para el Modelado de Procesos de Negocio (BPMO, del inglés Business Process Modelling Ontology), posibilita la anotación semántica en modelos de procesos de negocio con alto nivel de abstracción. BPMO incluye conceptos que describen el comportamiento de los procesos, actividades y datos organizacionales en un nivel semántico [3], [15], integrando conocimiento acerca del contexto organizacional, actividades del flujo de trabajo y SWS.

BPMO provee soporte para varias actividades de BPM, tales como modelado, ejecución, y análisis [15]. Utiliza varias técnicas de razonamiento y representación de conocimiento para permitir que el flujo de procesos de negocio pueda ser expuesto y compartido a través de descripciones semánticas; para referenciar datos y servicios de negocio anotados semánticamente; para incorporar datos heterogéneos a través de mapeo semántico; y para efectuar consultas usando un razonador o motor de inferencia.

En resumen, BPMO aprovecha las facilidades de las tecnologías de SWS para que en los procesos se puedan: 1) utilizar descripciones semánticas, 2) referirse a los datos y servicios semánticamente anotados mediante URI's, 3) incorporar datos heterogéneos mediante mapeo semántico, y 4) realizar consultas (queries) usando razonadores o motores de inferencia.

\section{CASO DE ESTUDIO}

Para facilitar una mejor comprensión de cómo funciona la SBPM, en esta sección se presenta un hipotético, pero realista, escenario de un proceso de gestión de pronóstico de demanda entre dos organizaciones basado en el escenario propuesto en [16]. Este proceso de negocio es modelado con el lenguaje BPMN, posteriormente son anotados semántica todos elementos contenidos el diagrama de procesos de negocio utilizando el framework WSMO y los conceptos de la ontología BPMO.

La Organización-A "Cliente" y la Organización-B "Proveedor" han establecido un acuerdo de colaboración con el propósito de mejorar sus beneficios y disminuir sus costos de operación. Estas empresas 
desean definir una colaboración B2B y los procesos de negocio colaborativos que deberían estar involucrados en la gestión de un pronóstico de demanda y un plan de suministro colaborativo.

El proceso de gestión de pronóstico de demanda colaborativo, soporta una negociación entre una organización que cumple el rol de cliente y otra organización que cumple el rol de proveedor para acordar un pronóstico de demanda. El proceso de negocio inicia con el cliente, que solicita al proveedor la realización de un pronóstico de demanda.

El documento de negocio contenido en el mensaje de solicitud transmite los datos a tener en cuenta en el pronóstico (por ejemplo: productos, horizontes de tiempos del pronóstico, etc.). El proveedor procesa la solicitud y puede responder aceptando o rechazando dicha solicitud. En el caso de que el rol proveedor acepte la propuesta, se compromete a realizar el pronóstico de demanda requerido; de lo contrario si el proveedor rechaza la propuesta, el proceso finaliza con una terminación de falla del sistema.

Cuando el proveedor acepta la solicitud, el cliente debe enviar el pronóstico de ventas de sus puntos de venta (POS) y los eventos y políticas de ventas planificadas, en forma paralela. Por último, con esta información, el proveedor genera un pronóstico de demanda, el cual es enviado al cliente, finalizando satisfactoriamente el proceso.

En la Fig. 2 se muestra el modelado del proceso de negocio del escenario presentado, utilizando el lenguaje BPMN, se detalla la interacción entre las organizaciones participantes a través del intercambio de mensajes, los cuales contienen los documentos de negocio que se envían o reciben. Las interacciones modeladas en el proceso de negocio representan la coreografía del proceso.
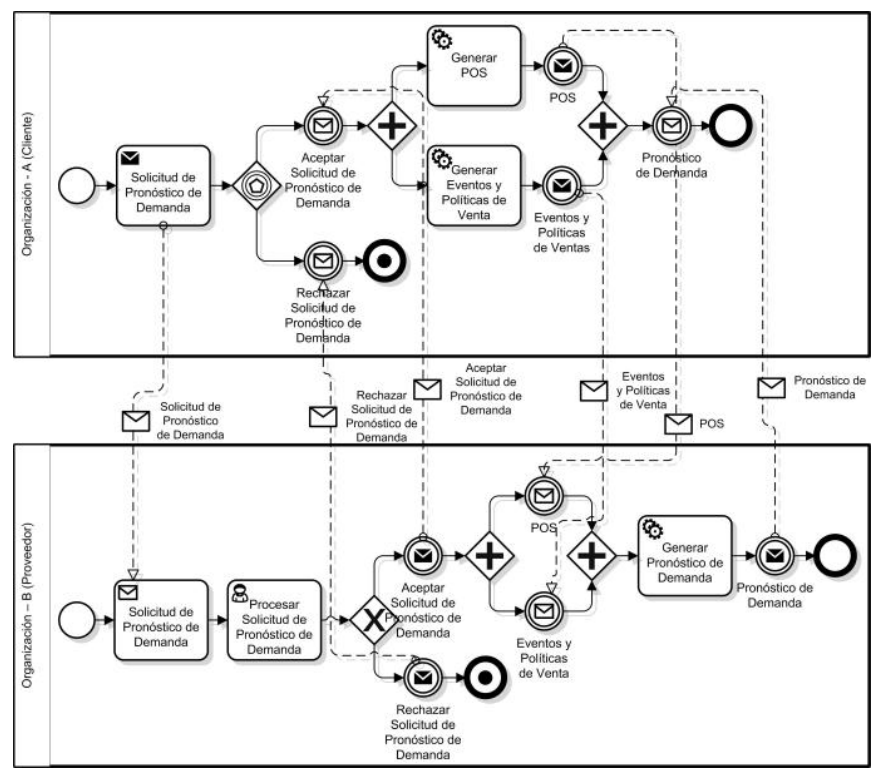

Fig. 2. Modelado del proceso de negocio colaborativo
En la fase de modelado del proceso de negocio, los elementos que componen el diagrama del proceso son anotados semánticamente, en el escenario modelado en la Fig. 2 las tareas de tipo servicio Generar POS y Generar Eventos y Políticas de Venta del contenedor (poo) Organización-A son anotadas con el servicio Web que habilitará su implementación. También, la tarea Procesar Solicitud de Pronóstico de Demanda del pool Organización- $B$ es anotada con el correspondiente servicio Web. En la Fig. 3 se muestra un extracto de la especificación generada del proceso de negocio modelado. Esta especificación se basa en los conceptos de la ontología BPMO.

La Fig. 3 presenta la estructura raíz de una tarea de tipo servicio, así como la instancia servicio (service_1368..._1187522882) con sus atributos, la cual corresponde a la tarea Eventos y Políticas de Venta. También, describe cual es la salida de la tarea o que servicio se utilizará para procesar los datos generados por la tarea, lo cual se muestra por medio del valor del atributo outputDescription, que contiene el valor http://148.237.71.34/server/name.

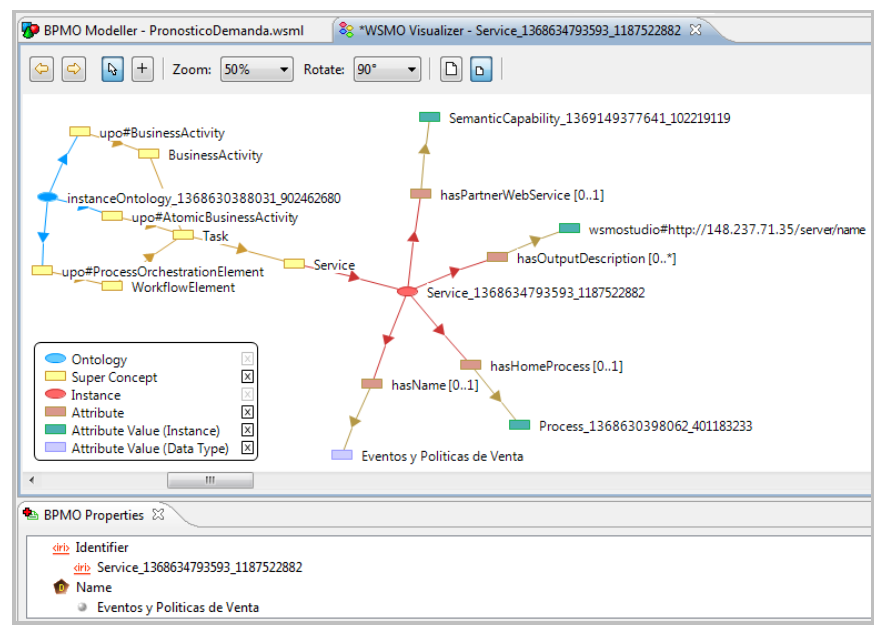

Fig. 3. Instancia de una tarea de servicio y sus atributos

Además, mediante el atributo PartnerWebService (Fig. 3) se define la relación con el servicio Web que habilitará la implementación de la tarea. En la Fig. 4 se muestra la instancia del concepto SemanticCapability mediante el cual se especifica el servicio Web que implementará la tarea. En este caso, el servicio Web definido corresponde a la tarea Eventos y Políticas de Venta, la relación entre esta instancia del servicio y la instancia de la tarea se genera a través del valor del atributo PartnerWebService (Fig. 3) y el identificador de la instancia SemanticCapability (Fig. 4).

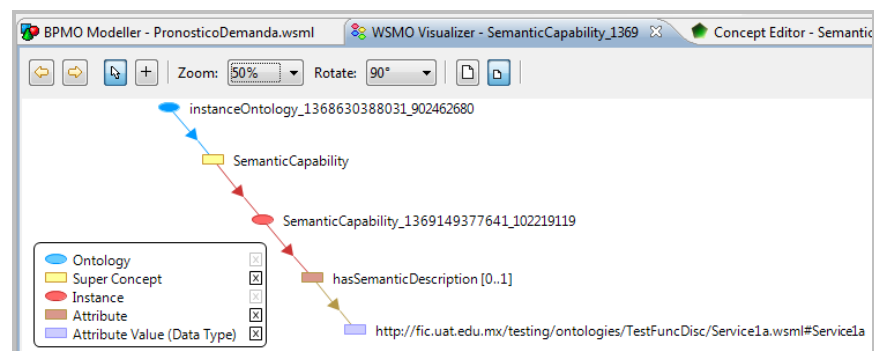

Fig. 4. Instancia del concepto capacidad semántica 
Un aspecto importante de la gestión de procesos de negocio semánticos es la capacidad que se tiene para realizar razonamiento automático sobre los modelos de los procesos de negocio. Con el objetivo de ejemplificar este aspecto, en la Fig. 5 se presenta una consulta realizada utilizando la herramienta del razonador IRIS [17] implementada mediante WSMO. La consulta realizada permite identificar todas las instancias de los elementos contenidos en el proceso que tengan definido tanto un servicio Web como una salida de la tarea. Además, identifica las instancias del concepto SemanticCapability y sus atributos relacionados a los elementos encontrados por la consulta ejecutada (en este caso, tareas de tipo servicio).

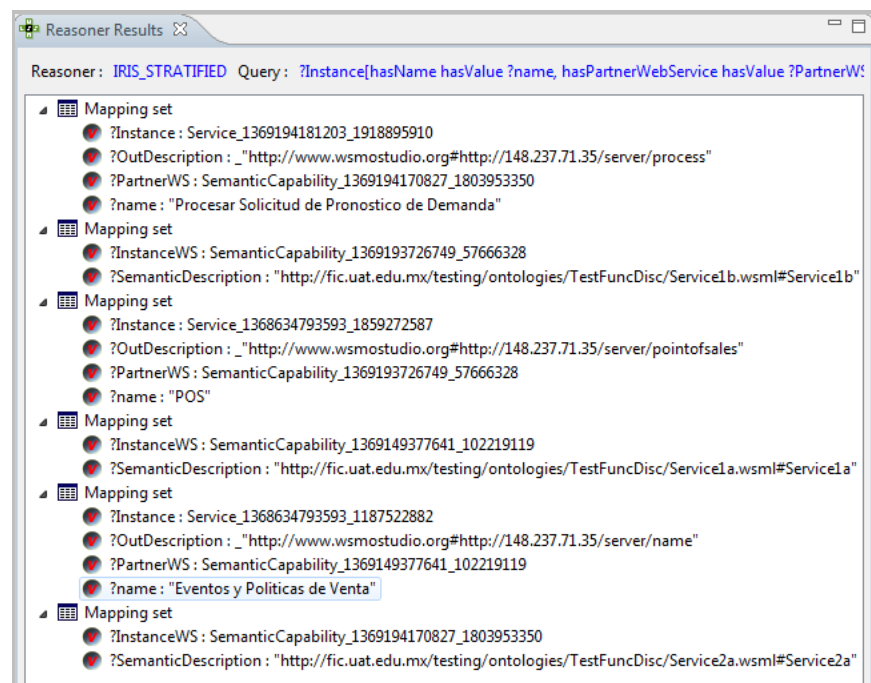

Fig. 5. Resultados generados por el razonador sobre las instancias del proceso de negocio semántico

\section{CONCLUSIONES}

La aplicación de la ontología BPMO, que combina conceptos de lenguaje BPMN y EPC, permite enriquecer el modelado conceptual de los procesos de negocio, habilitando la anotación semántica de los procesos mediante la herramienta WSMO. Lo cual conlleva grandes ventajas para los analistas de negocio y para los ingenieros de $\mathrm{Tl}$, permitiendo definir claramente los elementos del proceso mediante conceptos más cercanos al entorno de negocio (perspectiva de negocio) y especificando los servicios que permiten implementar tareas contenidas en el proceso. Esta especificación puede ser mediante un descubrimiento basado en semántica de fragmentos de procesos, lo cual permite disminuir tiempos de desarrollo y/o errores que se pueden presentar en etapas tempranas del desarrollo.

En el escenario presentado se puede observar la capacidad de la herramienta para ejecutar razonamiento automático identificando instancias del proceso o en su caso fragmentos de proceso que pueden ser reutilizados en otros procesos. Posteriormente, en la fase de análisis se puede ejecutar razonamiento sobre los logs generados en la fase de ejecución del proceso semántico, con lo cual se permite analizar posibles desviaciones y mejoras al proceso de negocio, aplicando técnicas de minería de procesos. Esta fase se encuentra dentro de los trabajos futuros que se tiene planeado enfrentar para poder realizar un análisis completo de la gestión de procesos de negocio semántico mediante la aplicación en diferentes dominios de implementación.

\section{REFERENCIAS}

[1] M. Weske. "Business Process Management. Concepts, Languages, Architectures”. Berlin: Springer, 2007, pp. 4-11.

[2] M. Hepp and R. Dumitru. "An Ontology Framework for Semantic Business Process Management". In Proceedings of the 8th Internationale Tagung Wirtschaftsinformatik 2007: eOrganisation: Service-, Prozess-, Market-Engineering, Karlsruhe, Germany, pp. 423-440, 2007.

[3] M. Dimitrov; A. Simov; S. Stein and M. Konstantinov. "A BPMO Based Semantic Business Process Modelling Environment". In Proceedings of the Workshop on Semantic Business Process and Product Lifecycle Management SBPM 2007, Innsbruck, Austria, pp. 101104, 2007.

[4] Object Management Group. "Business Process Modeling Notation (BPMN) specification version 2.0." Technical report, Object Management Group, 2011. [Online].

[5] J. de Bruijn; H. Lausen; A. Polleres and D. Fensel. "The web service modeling language WSML: an overview". In Proceedings of the 3rd European Semantic Web Conference, ESWC 2006, Budva, Montenegro, pp. 590604, 2006.

[6] D. Fensel; H. Lausen; J. de Brujin; M. Stollberg; D. Roman; A. Polleres and J. Domingue. "Enabling Semantic Web Services. The Web Service Modeling Ontology". Berlin: Springer, 2007.

[7] M.Hepp; F. Leymann; J. Domingue; A. Wahler and D. Fensel. "Semantic business process management: a vision towards using semantic Web services for business process management". In Proceedings of the IEEE International Conference on e-Business Engineering, ICEBE 2005. pp.535, 540, 2005.

[8] B. Wetzstein, Z. Ma, A. Filipowska, M. Kaczmarek, S. Bhiri, S. Losada, J.-M. Lopez-Cobo and L. Cicurel. "Semantic Business Process Management: A Lifecycle Based Requirements Analysis". In Proceedings of the Workshop on Semantic Business Process and Product Lifecycle Management SBPM 2007, Innsbruck, Austria, pp. 1-11, 2007.

[9] L. Cabral and J. Domingue. "Translating Semantic Web Service based business process models". In Proceedings of The IEEE Asia-Pacific Services Computing Conference (APSCC 2009), pp.1-6, 2009.

[10] D. Martin et al. OWL-S: "Semantic Markup for Web Services". W3C Member Submission, Online [Nov. 2004].

[11] D. Roman et al. "Web Service Modeling Ontology". Journal Applied Ontology, Vol. 1, No. 1, pp. 77-106, 2005.

[12] J. Kopecky, T. Vitvar, C. Bournez and J. Farrell. "SAWSDL: Semantic Annotations for WSDL and XML Schema". IEEE Internet Computing, Vol. 11, No. 6, pp. 60-67, 2007.

[13] D. Fensel and C. Bussler. "The Web Service Modeling Framework WSMF". Electronic Commerce Research and Applications, Vol. 1, No. 2, pp. 113-137, 2002. 
[14] A-W. Scheer, O. Thomas and O. Adam. "Process Modeling using Event-Driven Process Chains". In Process-Aware Information Systems: Bridging People and Software through Process Technology, (M. Dumas; W. M. P. van der Aalst and A. H. M. ter Hofstede, Eds.), Hoboken, NJ, USA: John Wiley \& Sons, Inc., 2005, pp. 116-146.

[15] L. Cabral, B. Norton and J. Domingue. "The business process modelling ontology". In Proceedings of the 4th International Workshop on Semantic Business Process Management, (SBPM-2009), Heraklion, Greece, pp. 916, 2009.
[16] P.D. Villarreal, I. Lazarte; J. Roa and O. Chiotti. "A Modeling Approach for Collaborative Business Processes Based on the UP-ColBPIP Language". In Business Process Management Workshops, (S. Rinderle-Ma; S. Sadiq and F. Leymann, Eds.), LNCS, Vol. 43. Berlin: Springer, 2010, pp. 318-329.

[17] B. Bishop and F. Fischer. "IRIS - Integrated Rule Inference System". In Proceedings of the Workshop on Advancing Reasoning on the Web: Scalability and Commonsense, ARea2008. pp. 18-32, 2008. 